\title{
Taxas de prevalência de hanseníase nas macrorregiões do estado de Mato Grosso: Datasus-Brasil
}

\begin{abstract}
RESUMO | Objetivo: analisar e comparar taxas de prevalência de hanseníase nas macrorregiões do estado de Mato Grosso a partir informações coletadas do Departamento de Informática do Sistema Único de Saúde (DATASUS) do Ministério da Saúde do Brasil. Método: estudo retrospectivo transversal onde analisou a taxa de prevalência no período de janeiro a dezembro de 2017. Os dados foram obtidos através do (DATASUS). Os valores foram comparados por análise de variância, testes de KolmogorovSmirnov e Levene, seguidos pelo teste post hoc de Tukey, com nível de significância em $p<0,05$. Resultados: Houve diferença estatisticamente significante entre as Macrorregiões do estado de Mato Grosso, Teles Pires, Vale do Peixoto, Vale do Arinos e Médio Araguaia, consideradas "hiperendêmicas". Conclusão: As taxas de prevalência de hanseníase encontradas neste estudo a partir da utilização da ferramenta DATASUS avaliando as macrorregiões do estado de Mato Grosso abrem perspectivas futuras para estudo epidemiológicos bem elaborados bem como a análise da qualidade dos serviços de saúde utilizados. A ferramenta DATASUS pode ser utilizada no planejamento de políticas públicas para a hanseníase.
\end{abstract}

Palavras-chaves: Epidemiologia; Hanseníase; Pesquisa Sobre Serviços de Saúde.

ABSTRACT | Background: Objective: To analyze and compare leprosy prevalence rates in the macroregions of the state of Mato Grosso from information collected from the Department of Informatics of the Unified Health System (DATASUS) of the Brazilian Ministry of Health. Method: a retrospective cross-sectional study analyzing the prevalence rate from January to December 2017. Data were obtained through (DATASUS). The values were compared by analysis of variance, Kolmogorov-Smirnov and Levene tests, followed by Tukey post hoc test, with significance level of $p<0.05$. Results: There was a statistically significant difference between the macroregions of the state of Mato Grosso, TelesPires, Peixoto Valley, Arinos Valley and Middle Araguaia, considered "hyperendemic". Conclusion: The prevalence rates of leprosy found in this study from the use of the DATASUS tool evaluating the macro-regions of the state of Mato Grosso open future perspectives for well-prepared epidemiological studies as well as the analysis of the quality of the health services used. DATASUS tool can be used in the planning of public policies for leprosy.

Keywords: Epidemiology; Health Services Research; Leprosy.

RESUMEN | Objetivo: analizar y comparar las tasas de prevalencia de lepra en los macrorregiones del estado de Mato Grosso a partir de la información recopilada del Departamento de Informática del Sistema Único de Salud (DATASUS) del Ministerio de Salud de Brasil. Método: estudio retrospectivo transversal que analiza la tasa de prevalencia de enero a diciembre de 2017. Los datos se obtuvieron a través de (DATASUS). Los valores se compararon mediante análisis de varianza, pruebas de KolmogorovSmirnov y Levene, seguidas de la prueba post hoc de Tukey, con un nivel de significación de $p<0,05$. Resultados: Hubo una diferencia estadísticamente significativa entre los macrorregiones del estado de Mato Grosso, Teles Pires, Peixoto Valley, Arinos Valley y medio Araguaia, consideradas "hiperendémicas". Conclusión: Las tasas de prevalencia de lepra encontradas en este estudio a partir del uso de la herramienta DATASUS que evalúa las macro regiones del estado de Mato Grosso abren perspectivas futuras para estudios epidemiológicos bien preparados, así como el análisis de la calidad de los servicios de salud utilizados. La herramienta DATASUS se puede utilizar en la planificación de políticas públicas.

Descriptores: Epidemiología; Investigación de servicios de salud; Lepra.

\section{Thiago Machado Pereira}

Cirurgião-dentista. Coordenador de Odontologia, Graduação em Odontologia, Faculdade do Norte de Mato Grosso, Guarantã do Norte (MT), Brasil. Doutor em Ciências Odontológicas Integradas.

\section{Fabiana Rezer}

Enfermeira. Professora, Graduação em Enfermagem, Faculdade do Norte de Mato Grosso. Guarantã do Norte (MT), Brasil. Mestre pelo Programa de Enfermagem Profissional no Processo do Cuidar em Saúde pelo Centro Universitário São Camilo, especialista em Nefrologia.

Recebido em: 09/12/2019

Aprovado em: 26/01/2020

\section{Wladimir Rodrigues Faustino}

Enfermeiro. Coordenador de Enfermagem, Graduação em Enfermagem, Faculdade do Norte de Mato Grosso. Guarantã do Norte (MT), Brasil. Mestre pelo Programa de Enfermagem Profissional da Gênese a Evolução do Cuidar pelo Centro Universitário São Camilo, especialista em UTI Adulto, Pediátrico, Neonatal e Nefrologia.

\section{Álvaro Henrique Borges \\ Cirurgião-dentista. Professor do Curso de Pós-Graduação em Odontologia da Universidade de Cuiabá. Cuiabá (MT), Brasil. Doutor em Endodontia.}

\section{INTRODUÇÃO}

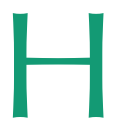
anseníase é definida como doença infecciosa crônica causada pelo Mycobacterium leprae ${ }^{1,2}$. Descoberta em 1873 pelo médico bacteriologista e dermatologista norueguês Armauer Hansen, afeta primariamente a pele e o sistema nervoso periférico ${ }^{3,4}$. É popularmente conhecida como lepra, sendo uma das doenças mais antigas que se tem conhecimento. A definição e classificação de casos utilizadas atualmente é baseada em critérios clínicos e bacteriológicos e diferencia os 
pacientes em: multibacilares, apresentando baciloscopia positiva associada a mais de 5 lesões cutâneas; e paucibacilares, apresentando baciloscopia negativa com ${ }^{2-5}$ lesões ou lesão única sem acometimento de nervos periféricos ${ }^{1-5}$. Este agravo de saúde é responsável por um olhar preocupante pelos profissionais de saúde, pois além de causadora de incapacidades físicas, causa estigma social, depressão e isolamento ${ }^{6}$. Se tratando de um problema de saúde pública em países de baixa e média renda, apesar da magnitude e do impacto na saúde, a hanseníase apresenta pouco investimento em pesquisa e desenvolvimento terapêutico ${ }^{7,8,9}$.

Em 2016, a partir de dados da Organização Mundial da Saúde (OMS) $)^{10,11}$, um total de 143 países relataram 214.783 casos de hanseníase, o que representa uma taxa de prevalência de 2,9 casos por 100 mil habitantes. No Brasil, no mesmo ano, foram notificados 25.218 casos novos, resultando em uma taxa de prevalência de 12,2/100.000 habitantes ${ }^{11}$. Esses números inserem o Brasil em um status de alta prevalência para hanseníase, sendo o segundo com o maior número de casos novos registrados no mundo. A região Centro-Oeste do Brasil apresenta taxa de prevalência de hanseníase de 40,04/100.000 habitantes, sendo o segundo índice de todas as regiões, atrás apenas da região Norte ${ }^{11}$. Mudanças socioeconômicas e expansão da Estratégia Saúde da Família, ocorridas nas últimas décadas no Brasil, influenciaram os determinantes sociais da saúde, consequentemente, a incidência de doenças infecciosas reduziu, porém, o impacto na hanseníase ainda não é totalmente claro ${ }^{11-13}$.

O estado brasileiro de Mato Grosso apresenta números preocupantes para casos de hanseníase ocupando a primeira posição na região Centro-Oeste com as maiores taxas de prevalência e incidência da doença, independen- te do ativo crescimento econômico e introdução do modelo de atenção à saúde pública nas últimas décadas no estado $^{11,12,13}$. Porém a literatura apresenta escassez de informações sobre a distribuição dos casos notificados e taxas de prevalência nas macrorregiões do estado de Mato Grosso ${ }^{3}$, justificando assim a aplicação de estudos epidemiológicos analisando possíveis focos deste agravo.

Dados de saúde pública, assim como, prevalência da hanseníase nas macrorregiões do estado de Mato Grosso, são acessíveis pela base de dados Departamento de Informática do Sistema Único de Saúde (DATASUS) ${ }^{14}$. Esta ferramenta apresenta facilidade no acesso e eficiência na obtenção de dados e informações em saúde pública, estando disponível para qualquer cidadão $^{15}$. O objetivo deste estudo foi analisar e comparar taxas de prevalência de hanseníase nas macrorregiões do estado de Mato Grosso no período compreendido entre janeiro e dezembro de 2017 a partir da ferramenta DATASUS. A hipótese nula avaliada foi de que não haveria diferença estatisticamente significante entre as taxas de prevalência de hanseníase nas macrorregiões analisadas.

\section{MÉTODO}

Esta pesquisa descritiva e transversal analisou e comparou a taxa de prevalência de hanseníase nas macrorregiões do estado de Mato Grosso no período compreendido entre janeiro e dezembro de 2017.

Os dados foram obtidos por meio de consultas nas bases de dados do Ministério da Saúde (DATASUS). O link http://datasus.saude.gov.br foi acessado dia 15 de abril de 2019. A pesquisa seguiu os indicadores: "acesso à informação"; "informações sobre saúde (TABNET)"; "Epidemiológicas e Morbidade"; "Casos de Hanseníase (SINAN)"; "Hanseníase". Em seguida o indicador "Geral - Indicadores epidemiológicos e operacionais de hanseníase, por ano diagnóstico Municípios/UF/Regiões/Brasil - 2017" foi acessado. Para esta pesquisa, foi selecionado o estado de Mato Grosso. As variáveis selecionadas foram: Macrorregião de saúde de notificação (Alto Tapajós, Araguaia Xingu, Baixada Cuiabana, Centro Norte, Garças Araguaia, Médio Araguaia, Médio Norte Mato-grossense, Noroeste Mato-grossense, Norte Araguaia Karajá, Norte Mato-grossense, Oeste Mato-grossense, Sudoeste Mato-grossense, Sul Mato-grossense, Teles Pires, Vale do Peixoto, Vale dos Arinos), e o campo taxa de prevalência (Hanseníase).

A tabulação foi feita por meio do Programa Tab para Windows - TABWIN (Ministério da Saúde, Brasília, Brasil) e transcritas em tabelas e gráficos usando o Microsoft Excel 16.23 - Office 3652019 (Microsoft Corp., Redmond, EUA). Os valores foram comparados por análise de variância e os testes de Kolmogorov-Smirnov e Levene seguidos pelo teste post hoc de Tukey. O nível de significância foi definido em $p<0,05$. Os valores foram estratificados de acordo com as classificações: "Hiperendêmico"; "Muito Alto"; "Alto"; e "Médio". Sendo que, estas classificações foram adotadas pelo próprio sistema de base de dados DATASUS. Todas as análises estatísticas foram realizadas usando SPSS versão 18.0 para Windows (SPSS Inc, Chicago, EUA). Essa pesquisa não foi submetida ao Comitê de Ética e Pesquisa com Seres Humanos, de acordo com a resolução 466/2012, por se tratar de dados de domínio público.

\section{RESULTADOS}

A taxa prevalência de hanseníase nas macrorregiões do estado de Mato Grosso no período compreendido entre janeiro e dezembro de 2017 foram apresentados na Tabela 1. As macrorre- 
giões do Teles Pires $(20,91)$, Vale do Peixoto (22,38), Vale dos Arinos $(28,29)$ e Médio Araguaia $(37,55)$ foram classificados como "Hiperendêmicas".

\begin{tabular}{|c|c|c|}
\hline Macrorregião de Saúde de Notificação & Taxa Prevalência & Classificação \\
\hline Sul Mato-grossense & $4,12 * a$ & Médio \\
\hline Norte Araguaia Karajá & $4,19^{a}$ & Médio \\
\hline Médio Norte Mato-grossense & $4,34^{a}$ & Médio \\
\hline Oeste Mato-grossense & $4,90^{\mathrm{a}}$ & Médio \\
\hline Noroeste Mato-grossense & $5,44^{b}$ & Alto \\
\hline Sudoeste Mato-grossense & $7,21^{b}$ & Alto \\
\hline Centro Norte & $7,56^{\mathrm{b}}$ & Alto \\
\hline Baixada Cuiabana & $8,59^{b}$ & Alto \\
\hline Araguaia Xingu & $11,63^{c}$ & Muito Alto \\
\hline Alto Tapajós & $14,11^{\mathrm{c}}$ & Muito Alto \\
\hline Norte Mato-grossense & $15,23^{c}$ & Muito Alto \\
\hline Garças Araguaia & $15,78^{c}$ & Muito Alto \\
\hline Teles Pires & $20,91^{d}$ & Hiperendêmico \\
\hline Vale do Peixoto & $22,38^{d}$ & Hiperendêmico \\
\hline Vale dos Arinos & $28,29^{d}$ & Hiperendêmico \\
\hline Médio Araguaia & $37,55^{d}$ & Hiperendêmico \\
\hline Total do Estado & $10,86^{c}$ & Muito Alto \\
\hline
\end{tabular}

* Letras diferentes indicam diferenças estatisticamente significantes. Fonte: http://datasus.saude.gov.br acessado em 15 de abril de 2019.

\section{DISCUSSÃO}

Os valores de taxa de prevalência de hanseníase, apresentados em ordem decrescente, foram encontrados na macrorregião do: Médio Araguaia $(37,55)$; Vale dos Arinos (28,29); Vale do Peixoto $(22,38)$; Teles Pires (20,91); Garças Araguaia $(15,78)$; Norte Mato-grossense (15,23); Tapajós $(14,11)$; Araguaia Xingu (11,63); Baixada Cuiabana $(8,59)$; Centro Norte $(7,56)$; Sudoeste Mato-grossense $(7,21)$; Noroeste Mato-grossense (5,44); Oeste Mato-grossense (4,90); Médio Norte Mato-grossense $(4,34)$; Norte Araguaia Karajá $(4,19)$; A macrorregião do Sul Mato-grossense $(4,12)$. A hipótese nula foi rejeitada uma vez que houve diferenças estatisticamente significativas entre as macrorregiões analisadas.

Esta pesquisa utilizou o DATASUS como fonte de dados devido ao seu simplificado acesso e sua eficiência na obtenção de informações em saúde ${ }^{15}$. À dem ser aplicadas em todos os níveis de gestão de saúde publica, melhorando o processo de sua administração.

As macrorregiões do Médio Araguaia, Vale dos Arinos, Vale do Peixoto e Teles Pires apresentaram valores de taxa de prevalência superiores aos valores médios apresentados pelo estado de Mato Grosso em sua totalidade. Este achado indica necessidade de realização de estudos epidemiológicos que possam aprofundar estes dados de áreas de alto risco, no intuito de gerar monitoramento e aplicação de ações emergenciais de políticas de saúde pública, visando redução das taxas de prevalência de hanseníase. A região Centro-Oeste apresenta a segunda maior taxa de prevalência de Hanseníase no Brasil, e o estado de Mato Grosso apresenta a maior taxa de prevalência do Centro-Oeste $^{4}$. Estes valores justificam a necessidade de análise aprofundada da distribuição da Hanseníase no estado de Mato Grosso, visto que a literatura apresenta escassez de dados comparando suas diferentes macrorregiões e nenhuma hipótese, relacionada a estas diferentes taxas de prevalência, pode ser confirmada por este estudo.

Apesar do avanço no controle da hanseníase no Brasil, alcançado tanto pelas ações preventivas quanto pela indicação de tratamentos poliquimioterápicos, é relatado que o uso de múltiplas drogas requer maior treinamento dos profissionais envolvidos, bem como maior compreensão dos pacientes, dificultando assim a adesão do paciente ao tratamento ${ }^{2,3}$. Entretanto, a hanseníase permanece como problema de saúde no estado de Mato Grosso, e a alta taxa de prevalência também pode estar relacionada, entre outros fatores, à ocorrência de recidivas. Ferreira et al. ${ }^{13}$ concluíram que a ocorrência de recidiva pode estar associada com condições de moradia, hábitos de vida, organização dos serviços de saúde dos municípios e esquemas tera- 
pêuticos. Como medidas de prevenção da recidiva, cabe aos profissionais de saúde oferecer orientações adequadas aos pacientes, bem como garantir a regularidade do tratamento. Outro ponto desafiador é o real conhecimento das taxas de prevalência da hanseníase, visto que os indicadores ainda permanecem sendo desenvolvidos por meio de estimativas ${ }^{16-19}$. Sendo assim, a real prevalência da hanseníase pode ser resultado da totalização da prevalência conhecida com a prevalência oculta a ser estimada $^{17,18}$. No entanto, segundo a Organização Mundial de Saúde não existe método eficaz que possibilite estimar com precisão a prevalência real da hanseníase tanto ao nível nacional quanto regional ${ }^{17}$.

A vigilância das regiões endêmicas é de extrema importância, pois a exposição de indivíduos pode resultar no aumento do risco de infecção ${ }^{1}$. Além disso, disparidades regionais implicam na sua distribuição, onde a grande extensão do território de Mato Grosso também dificulta o processo de rastreio dos casos para medidas preventivas ${ }^{17}$. Com isso, a presente pesquisa corrobora com pesquisas anteriores em relação as regiões irregulares, com diferentes indicadores econômicos e diferentes níveis de atenção à saúde 2,3,13,17-19. Traçar um perfil epidemiológico da hanseníase relacionando indicadores como IDH, envolvimento com outras condições sistêmicas, características ambientais e pobreza, possibilitaria, além de detectar as áreas de risco, indicar instrumentos de prevenção, visando reduzir a taxa endêmica da doença ${ }^{17-21}$.

Neste presente estudo, fica destacado o uso de informações obtidas na base de dados do Ministério da Saúde, sendo que, tais dados dependem da atualização on-line do sistema pelos gestores municipais, permitindo implementar medidas preventivas. Como implicações na prática clínica, este estudo poderá contribuir no desenvolvimento de medidas de prevenção nas regiões supracitadas, visando manter o ritmo de organização do sistema público de saúde em prol da redução dos casos e fu- tura erradicação da hanseníase. Estimativas sobre taxas de prevalência respaldariam a criação de estratégias para a detecção de casos, identificação de focos de transmissão e consequente eliminação da doença ${ }^{18-20}$. Portanto as diferentes taxas de prevalência de hanseníase encontradas neste estudo a partir da utilização da ferramenta DATASUS avaliando as macrorregiões do estado de Mato Grosso abrem perspectivas futuras para estudo epidemiológicos bem elaborados bem como a análise da qualidade dos serviços de saúde utilizados.

\section{CONCLUSÃO}

As macrorregiões do Teles Pires, Vale do Peixoto, Vale dos Arinos e Médio Araguaia apresentaram maiores valores de taxa de prevalência de hanseníase em relação às demais macrorregiões do estado de Mato Grosso, sendo consideradas hiperendêmicas. A ferramenta DATASUS pode ser utilizada no planejamento de políticas públicas para a hanseníase.

\section{Referências}

1. Prevedello FC, Mira MT. Leprosy: a genetic disease? An Bras Dermatol. 2007;82(5):451-9.

2. Freitas BHBM, Cortela DDCB, Ferreira SMB. Trend of leprosy in individuals under the age of 15 in Mato Grosso (Brazil), 2001-2013. Rev Saude Pub. 2017;51:28.

3. Queiróz ML, Scatena JHG. Temporal and spatial distribution of Hansen's disease in the state of Mato Grosso, from 1996 to 2007. Cad Saude Colet. 2009;17(1):145-161.

4. Silva CLM, Fonseca SC, Kawa H, Palmer DOQ. Spatial distribution of leprosy in Brazil: a literature review. Rev SocBrasMed Trop. 2017;50(4):439-449.

5. Cruz RCDS, Bührer-Sékula S, Penna MLF, Penna GO, Talhari S. Leprosy: current situation, clinical and laboratory aspects, treatment history and perspective of the uniform multidrug therapy for all patients. An BrasDermatol. 2017;92(6):761-73.

6. Scollard DM, Adams LB, Gillis TP, Krahenbuhl JL, Truman RW, Williams DL. The continuing challenges of leprosy. ClinMicrobiol Rev. 2006;19(2):338-81.

7. Goulart IM, Goulart LR. Leprosy: diagnostic and control challenges for a worldwide disease. Arch Dermatol Res. 2008;300(6):269-90.

8. Rodrigues LC, Lockwood DNj. Leprosy now: epidemiology, progress, challenges, and research gaps. Lancet Infect Dis. 2011;11(6):464-70.

9. Ginsberg AM. Leprosy research - Setting priorities and facilitating collaborations: A personal perspective. Lepr Rev. 2000;71:183-7.

10. Miranzi SSC, Pereira LHM, Nunes AA. Epidemiological profile of leprosy in a Brazilian municipality between 2000 and 2006. Rev Soc Bras Med Trop. 2010;43(1):62-7.

11. Ministério da Saúde (BR), Secretaria de Vigilância em Saúde. Situação epidemiológica da hanseníase no Brasil: análise de indicadores selecionados na última década e desafios para eliminação. BolEpidemiol. 2013
12. Ramos JMH, Souto FJD. Disability after treatment among leprosy patients in Várzea Grande, State of Mato Grosso. Rev Soc Bras Med Trop. 2010;43(3):293-7.

13. Ferreira $S M B$, Ignotti $E$, Gamba MA. Factors associated to relapse of leprosy in Mato Grosso, Central-Western Brazil. Rev Saude Pub. 2011;45(4):1-8. 14. Departamento de Informática do Sistema Único de Saúde (DATASUS) [site da Internet]. [Acesso em 15/04/2019]. Disponível em: http://datasus.saude. gov.br/.

15. Pereira TM, Borges AH, Costa LMAS, Oliveira D, Volpato LER. Provision of endodontics in specialized public centers for dental care in the Center-West of Brazil. Rev ABENO. 2018;18(2):55-61.

16. Lima AC, Januário MC, Lima PT, Silva WM. DATASUS: o uso dos Sistemas de Informação na Saúde Pública. REFAS - Revista Fatec Zona Sul. 2015;1(3):16-31.

17. Ignotti E, Rodrigues AM, de Andrade VLG, Valente JG. Application of estimation methods for the hidden prevalence of leprosy in the State of Mato Grosso. Rev Bras Epidemiol. 2004;7(2):155-66.

18. Magalhães MCC, dos Santos ES, de Queiroz ML, de Lima ML, Borges RCM, Souza M, Ramos AN. Migration and Hansen's disease in Mato Grosso. Rev Bras Epidemiol. 2011;14(3):386-97.

19. Munhoz-Jr S, Fontes JF, Meirelles SMP. Evaluation of the program for leprosy control in counties of Mato Grosso State, Brazil. Rev Saude Pub. 1997;31(3):282-7.

20. Soprani dos Santos A, Silveira de Castro D, Falqueto A. Riskfactors for Leprosytransmission. Reben. 2008;61:738-743.

21. Matos FZ, Aranha AMF, Borges AH, Pedro FLM, Raslan SA, Hamida F, Veiga K, Porto AN. Can different stages of leprosy treatment influence the profile of oral health? Oral status in leprosy. Med Oral Patol Oral Cir Bucal. 2018;23:e376-83. 